\title{
Arteriosclerosis, Thrombosis, and Vascular Biology
}

\section{EDITORIAL}

\section{The Mast Cell}

\section{A Novel Actor in Cardiac Microvessel Dysfunction}

Ilze Botel

I $n$ the last decade, the mast cell, a potent immune cell mainly known for its function in host defense responses and for its contribution to allergies, has gained attention as effector cell in cardiovascular and metabolic diseases such as atherosclerosis, vein graft disease, diabetes, and obesity. ${ }^{1}$ Mast cells have been reported to contribute to the underlying proinflammatory pathways involved in these diseases, but the mast cell can also directly affect the surrounding tissue by the release of proteases and growth factors.

\section{See accompanying article on page e193}

In a recent study by Guimbal et al ${ }^{2}$ entitled "Mast Cells Are the Trigger of Small Vessel Disease and Diastolic Dysfunction in Diabetic Obese Mice," mast cells are now identified as a causal player in cardiac microvessel disease in leptin-receptor deficient (Lepr ${ }^{\mathrm{db} / \mathrm{db}}$ ) mice, an experimental model of diastolic dysfunction associated with heart failure with preserved ejection fraction. After confirming the presence of cardiac microvessel disease and diastolic dysfunction in this mouse model, the authors show via RNA sequencing analysis extensive inflammation in the heart, with a prominent upregulation of mast cell-related genes. Indeed, in the hearts of the Lepr ${ }^{\mathrm{db}}$ / $\mathrm{db}$ mice, the activated mast cell content was increased compared with the control mice. Being the most potent mast cell activation mechanism, the IgE-FceRI (Fc epsilon receptor 1) pathway is suggested to be involved, but despite elevated circulating IgE levels in the Lepr ${ }^{\mathrm{db} / \mathrm{db}}$ mice, the percentage of cardiac mast cell that had $\operatorname{lgE}$ bound to their surface did not differ between the groups.
Although this may suggest that other mast cell activation mechanisms, such as via complement receptors are at play here, the IgE-FCERI pathway should not be excluded yet based on IgE binding only. IgE binding to the FCERI only sensitizes the mast cell, whereas binding of an antigen to IgE molecules on the FceRI results in mast cell activation and degranulation. ${ }^{3}$ Detailed analysis of the cardiac mast cell fraction with respect to activation status and IgE-antigen binding will provide more evidence regarding the contribution of the IgE-FceRI pathway to this disease. In addition, elucidation of the pathways that have led to the striking increase in circulating lgE levels in these mice may provide more underlying mechanistic insights. For example, the elevated B-cell numbers shown in this study may point towards an increase in antibodyproducing B cells upon cardiac dysfunction. Further characterization of $\mathrm{B}$-cell subsets will thus provide valuable information regarding the underlying disease processes in the Lepr ${ }^{\mathrm{db} / \mathrm{db}}$ mice. This is also relevant given the fact that in a recent study a role for the lgE-FceR1 pathway in heart failure was established. ${ }^{4}$ Although the heart failure mouse models used in the study by Zhao et $\mathrm{al}^{4}$ are not directly comparable to the Lepr ${ }^{\mathrm{db} / \mathrm{db}}$ model of diastolic dysfunction, circulating lgE levels were increased upon induction of heart failure as well, and interestingly, blocking of the lgE-FceR 1 pathway reduced cardiac remodeling in vivo. In addition, depletion of the FceR1 directly improved cardiomyocyte and cardiac fibroblast function in vitro, suggesting that lgE antibodies may not only have direct effects on mast cells but also affect nonmyeloid cells in the heart, thereby contributing to a reduced cardiac function.

Nonetheless, Guimbal et $\mathrm{al}^{2}$ provide compelling evidence that the mast cell, or more specifically mast

Key Words: Editorials $\boldsymbol{\|}$ atherosclerosis $\boldsymbol{\|}$ heart failure $\boldsymbol{\|}$ inflammation $\boldsymbol{\|}$ mast cell $\boldsymbol{\|}$ microvessel

\footnotetext{
The opinions expressed in this article are not necessarily those of the editors or of the American Heart Association. Correspondence to: Ilze Bot, PhD, Einsteinweg 55, 2333 CC, Leiden, the Netherlands. Email i.bot@lacdr.leidenuniv.nl For Disclosures, see page 1338. (C) 2021 American Heart Association, Inc.

Arterioscler Thromb Vasc Biol is available at www.ahajournals.org/journal/atvb
} 
cell-derived histamine, contributes to cardiac microvessel disease in Lepr ${ }^{\mathrm{db} / \mathrm{db}}$ mice by showing that both mast cell stabilization with cromolyn and blockade of the $\mathrm{H} 1$ receptor by the $\mathrm{H} 1$-antagonist cetirizine reduce microvessel diameter in the heart, while also reducing vascular leakage. Total vessel density did not differ between the control and treatment groups, suggesting that microvessel functionality, but not microvessel growth, is primarily affected by the activated mast cells in the heart. Similar findings have been reported in an earlier study in a mouse model of hypoxia, in which local mast cell activation in the ischemic hindlimb enhanced collateral diameter, but did not affect collateral number. ${ }^{5}$ Also in human advanced atherosclerosis, mast cell numbers were associated with intraplaque microvessels that are prone to become leaky and contribute to intraplaque hemorrhage. ${ }^{6}$ Together, these studies demonstrate that mast cells, when activated, can have severe impact on microvessel function in different disease processes.

Interestingly, both cromolyn and cetirizine reduced the inflammatory response by reducing the number of $\mathrm{CD}_{45^{+}}$cells in the cardiac tissue, which suggests that histamine is the predominant mast cell mediator responsible for the observed proinflammatory effects. However, mast cells not only secrete histamine but can, depending on the activation stimulus, secrete mast cell-specific proteases, such as chymase and tryptase, leukotrienes, and a vast amount of proinflammatory cytokines that enhance the inflammatory response. Release of such cytokines likely also contributes to cardiac microvessel dysfunction as evident from the increase in cardiac inflammation. Therapeutically, it may thus be more beneficial to intervene in mast cell activation pathways rather than blocking the effects of a single mast cell mediator. Systemic blockade of mast cell activation may however have its drawbacks as well, as mast cells do have a valuable function in the defense against pathogens. Therefore, in any disease in which mast cells have been implicated, it is of importance to elucidate whether a disease-specific trigger for mast cell activation exists and assess whether this can be specifically and therapeutically targeted. Yet, in this study, both mast cell stabilization and $\mathrm{H} 1$ receptor antagonism appear promising as a therapeutic strategy in cardiac microvessel disease and diastolic dysfunction in vivo, rendering validation in patients suffering from heart failure with preserved ejection fraction warranted.

\section{ARTICLE INFORMATION}

\section{Affiliation}

Division of BioTherapeutics, Leiden Academic Centre for Drug Research, Leiden University, the Netherlands.

\section{Disclosures}

I. Bot is an Established Investigator of the Dutch Heart Foundation (2019T067).

\section{REFERENCES}

1. Shi GP, Bot I, Kovanen PT. Mast cells in human and experimental cardiometabolic diseases. Nat Rev Cardiol. 2015;12:643-658. doi: 10.1038/nrcardio. 2015.117

2. Guimbal S, Cornuault L, Rouault P, Hollier P-L, Chapouly C, Bats M-L, Imbault J, Gadeau A-P, Couffinhal T, Renault M-A. Mast cells are the trigger of small vessel disease and diastolic dysfunction in diabetic obese mice. Arterioscler Thromb Vasc Biol. 2021;41:e193-e207. doi: 10.1161/ATVBAHA.121.315900

3. Galli SJ, Borregaard N, Wynn TA. Phenotypic and functional plasticity of cells of innate immunity: macrophages, mast cells and neutrophils. Nat Immunol. 2011;12:1035-1044. doi: 10.1038/ni.2109

4. Zhao H, Yang H, Geng C, Chen Y, Pang J, Shu T, Zhao M, Tang Y, Li Z, Li B, et al. Role of IgE-FcepsilonR1 in pathological cardiac remodeling and dysfunction. Circulation. 2020. doi: 10.1161/CIRCULATIONAHA.120.047852.

5. Bot I, Velden DV, Bouwman M, Kröner MJ, Kuiper J, Quax PHA, de Vries MR. Local mast cell activation promotes neovascularizationCells. 2020;9:701. doi: 10.3390/cells9030701

6. Willems S, Vink A, Bot I, Quax PH, de Borst GJ, de Vries JP, van de Weg SM, Moll FL, Kuiper J, Kovanen PT, et al. Mast cells in human carotid atherosclerotic plaques are associated with intraplaque microvessel density and the occurrence of future cardiovascular events. Eur Heart J. 2013;34:36993706. doi: 10.1093/eurheartj/eht186 